\title{
Response-repetition costs in task switching: How they are modulated by previous-trial response-category activation
}

\author{
Kai Robin Grzyb *, Ronald Hübner \\ Universität Konstanz, Germany
}

PSycINFO codes:

2346 Attention

2343 Learning \& Memory

Keywords:

Response repetition

Task switching

Response inhibition

\begin{abstract}
A B S T R A C T
A common finding is that there are response-repetition (RR) costs under task switching. Moreover, when the stimulus on the previous trial was congruent then RR costs are usually larger than when it was incongruent. This effect of the previous trial has been explained by assuming that a response category is generally inhibited after the execution of its corresponding response on the previous trial and that the amount of inhibition depends on the activation of the response category. However, up to now it was open which property of the response-category activation on the previous trial is crucial: the absolute activation of the correct response category or the activation difference between the alternative response categories. To differentiate between these two possibilities we compared RR costs after congruent, neutral, and incongruent trials. In two experiments we found similar RR costs after congruent and neutral trials, whereas the RR costs were smaller after incongruent trials. These results support the hypothesis that the amount of response inhibition is determined by the activation differences between the alternative response categories on the previous trial.
\end{abstract}

\section{Introduction}

A frequently observed phenomenon in the area of task switching are response-repetition (RR) costs, i.e. the result that responding is slower and/or less accurate when the response from the previous trial repeats, compared to when it shifts (Kiesel et al., 2010; Rogers \& Monsell, 1995). Interestingly, the costs occur mainly on taskswitch trials. To explain these effects, several theories have been proposed (Altmann, 2011; Hübner \& Druey, 2006; Kleinsorge, 1999; Meiran, 2000; Schuch \& Koch, 2004), of which the response-inhibition account (Cooper \& Mari-Beffa, 2008; Hübner \& Druey, 2006) seems currently to be the most successful. In the present study we focused on this theory and tried to resolve an open question concerning one of its assumptions.

According to the response-inhibition model there is a risk that residual response activation from the previous trial carries over into the current trial. This would result in a bias in favor of the previous response, which would increase the risk of a perseveration error. Therefore, it is assumed that, in order to prevent such errors, the last response is generally inhibited after response execution (Hübner \& Druey, 2006). This inhibition of the previous response then produces costs for response repetitions on the current trial. The frequent result that no costs are observed on task-repetition trials (but see e.g., Cooper \& Marí-Beffa, 2008; Steinhauser \& Hübner, 2006) is explained

* Corresponding author at: Universität-Konstanz, Fachbereich Psychologie, Fach D29, D-78457 Konstanz, Germany. Tel.: + 49 7531/88 3153; fax: +49 7531/88 3017

E-mail address: KaiRobin,Grzyb@uni-konstanz,de (K.R. Grzyb). by the fact that on these trials other features of the previous task, e.g. the stimulus category or even the stimulus, also repeat, and that the positive effects of these repetitions outweigh the costs of a repeating response (see also Altmann, 2011). These assumptions imply that the pure effects of response repetition can only be observed on task-switch trials.

It follows from the response-inhibition account that the inhibition varies with the extent of response activation, because - without adaptive inhibition - a higher activation would result in higher residual activation which would increase the perseveration risk. This variation in inhibition should be reflected by a corresponding variation in RR costs on the next trial. This is exactly what has been observed (e.g. Druey \& Hübner, 2008b; Hübner \& Druey, 2006). Druey and Hübner, for instance, had their participants to judge the magnitude (smaller or larger than five) or parity (odd or even) of two subsequently presented numerals on each trial (Druey \& Hübner, 2008b, Experiment 1). A pre-cue informed about which judgment type was required for the first numeral and whether the same or the other judgment had to be performed for the second numeral. This two-task paradigm allowed a rather direct observation of the influence of the first task on the performance of the second one. In line with the responseinhibition account, it turned out that RR costs increased after responses to congruent stimuli, compared to RR costs after incongruent stimuli. Congruent and incongruent stimuli were numerals whose features activated the same response or opposite ones, respectively. For instance, if 'odd' and 'smaller than five' are mapped onto a 'left' response (e.g. require to press a left button), whereas 'even' and 'greater than five' are mapped onto a right response, then the numeral 3 is 
congruent and the numeral 7 is incongruent. Therefore, Druey and Hübner (2008b) assumed that congruent stimuli activate the correct response to a larger extent than incongruent ones. Hence, they reasoned that the observed modulation of RR costs by the congruency on the previous trial was due to a corresponding variation of response activations.

That the response activation on the previous trial is a crucial factor for RR costs is also supported by results of Druey and Hübner (2008b) showing that these costs can not only be manipulated by varying the congruency on the previous trial but also by varying the spatial compatibility on the previous trial, i.e. the overlap of spatial response features with spatial stimulus features (cf. Hommel, 2010; Simon, 1969). Moreover, Druey and Hübner (2008b) could show that the congruency and the spatial compatibility on the previous trial produce additive effects on RR costs.

\subsection{The aim of the present study}

Taken together, the considered results suggest that the response activation on the previous trial systematically affects RR costs on the current trial. However, it is still open exactly which properties of the response activation on the previous trial are actually relevant in this respect. Therefore, the goal of the present study was to investigate the origin of this previous-trial effect. In order to derive specific hypotheses we made the widely accepted assumption that response selection proceeds by continuously accumulating evidence from the stimulus at a certain rate in favor of the one or the other response. Usually the rate of evidence accumulation is conceptualized as the difference between the evidence provided by the stimulus for the individual responses. If the threshold for one of the responses is reached, then the corresponding motor response is triggered (c.f. Ratcliff, 1978; Ratcliff \& Rouder, 1998). Because it is reasonable to assume that the threshold remains constant across randomly varied conditions within a block of trials, the accumulated evidence at the time the response is selected is more or less constant across trials. Consequently, the amount of accumulated evidence in favor of the selected response cannot explain effects of factors such as stimulus congruency. This theoretical view is supported by results showing that activations of the motor system have no simple relation to the congruency of stimuli (Mattler, 2005). Likewise, the amount of accumulated evidence cannot account for differences in RR costs as observed by Druey and Hübner (2008b).

Thus, the only quantity of the response-selection system that can be related to the size of response inhibition is the activation of the response alternatives produced by the evidence provided by the stimulus. Critically, different from the accumulated evidence at the time of response selection, the evidence provided by the stimulus can vary across trials depending on the experimental conditions. Accordingly, our reasoning does not make any specific assumption of how evidence is accumulated to select a response. We merely assume that the provided evidence for the responses is reflected by the activation of corresponding central response representations.

The relevance of the activation of central response representations for our objective becomes apparent in the fact that RR effects are observed even if only the response category repeats (e.g., "left") from one trial to the next instead of the corresponding effector. This has been shown in studies using different output channels for the different task sets but with overlapping response categories (e.g., responding "left" with the left middle finger vs. responding "left" with the right index finger, Druey \& Hübner, 2008a; e.g., vocalizing "left" vs. pressing a left button, Schuch \& Koch, 2004). Moreover, Druey and Hübner (2008b) also used different effector sets for the different tasks. Consequently, not only the general RR effect but also the previous-trial effect is independent of effector repetitions. Thus, it is actually the response-category activation rather than the response activation that seems to determine the amount of RR costs on the subsequent trial. Therefore, throughout this paper, we will use the terms 'response-category activation' and, accordingly, 'responsecategory inhibition' to emphasize this distinction. However, this does not differentiate our account from previous response-inhibition accounts (e.g., Druey \& Hübner, 2008b) which, for reasons of simplicity, used both terms ('response' and 'response category') interchangeably.

Given these considerations, there are two possible properties of the response-category activation that could account for the modulation of RR costs. First, it is conceivable that the size of responsecategory inhibition is determined by the difference between the activations of the response categories. To consider an example, assume that the stimulus activates the category of the correct response by 0.7 and that of the wrong response by 0.4 (cf. Fig. 1A, incongruent case). Accordingly, the difference between these activations, which is also the rate at which evidence in favor of the correct response is accumulated over time, is 0.3 . We will call this idea the activationdifference hypothesis. Alternatively, it is also conceivable that responsecategory inhibition is determined by the absolute activation of the category of the triggered response. For our example the relevant value would be 0.7 (in case of a correct response). We will call this idea the absolute-activation hypothesis.

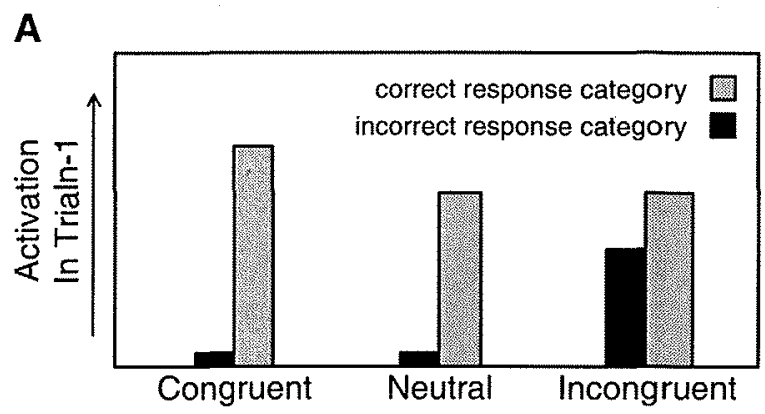

B

\section{Prediction by the absolute-activation hypothesis}

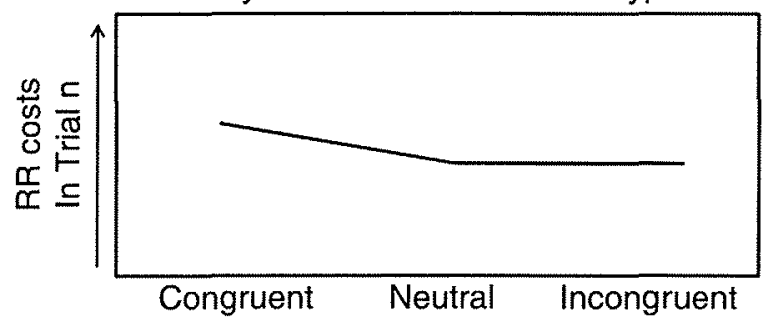

C

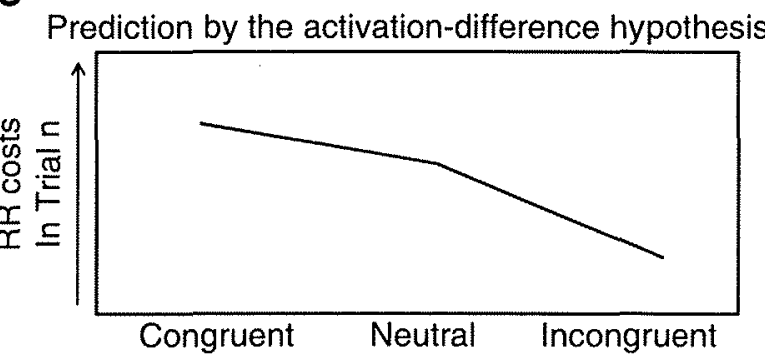

Fig. 1. Panel A: Schematic activation of the correct and incorrect responses categories for congruent. neutral, and incongruent stimuli in trial $n-1$. Note that the bars indicate activation that corresponds to the evidence provided by the stimulus. They do not indicate accumulated evidence (e.g. accumulated evidence at the time of response selection). Panels B and C: Hypothetical RR costs (repetitions minus shifts) in trial $n$ for three different conditions under the hypothesis that the size of response-category inhibition depends (B) on the absolute activation of the correct response category or ( $\mathrm{C}$ ) on the activation difference of the alternative response categories in trial $n-1$, 
Unfortunately, the available data did not allow us to differentiate between these two hypotheses. One reason was that studies investigating RR effects included only congruent and incongruent stimuli. To see why this selection is insufficient for answering our question, consider Fig. 1A, in which hypothetical activations of categories for correct and incorrect responses are shown for different congruency conditions. As can be seen, for congruent stimuli, the absolute activation of the correct response category is relatively high, because taskrelevant as well as task-irrelevant features of these stimuli activate the correct response category. Accordingly, the activation of the wrong response category is rather low. The situation is different for incongruent stimuli. In this case, only task-relevant features activate the correct response category. Consequently, its activation is smaller, compared to congruent stimuli. Moreover, there is also some activation of the wrong response category caused by task-irrelevant stimulus features. If we compare the activations of the correct response category for the two stimulus types, then they are higher for congruent than for incongruent stimuli. This difference corresponds to the absolute-activation hypothesis, and could explain the previous-trial effect of RR costs. However, as can also be seen in Fig. 1A, a similar relation holds with respect to the activation difference between the correct and the wrong response category. This difference is also higher for congruent than for incongruent stimuli, and corresponds to the activation-difference hypothesis.

These considerations show that one cannot differentiate between the absolute-activation hypothesis and the activation-difference hypothesis by simply comparing the RR costs after congruent with those after incongruent trials. Fortunately, the two hypotheses can be differentiated if we also include neutral stimuli in an experiment, i.e. stimuli whose irrelevant features activate neither the correct nor the wrong response category. In this case, the absolute activation of the correct response category should be larger for congruent than for neutral stimuli, but the absolute activation should not differ between neutral and incongruent stimuli. In contrast, the activation difference should be larger for neutral than for incongruent stimuli, and slightly larger for congruent than for neutral stimuli. Thus, by considering the response-category activations produced by congruent, neutral, and incongruent stimuli we can derive different predictions for the two hypotheses, which are illustrated in Fig. $1 B$ and $C$, respectively. As can be seen, the critical comparison is that between neutral and incongruent stimuli. The absolute-activation hypothesis predicts no difference between the two conditions (see Fig. 1B), whereas the activation-difference hypothesis predicts larger RR costs after neutral than after incongruent trials (see Fig. 1C).

\section{Experiment 1}

To test whether the absolute-activation hypothesis or the activation-difference hypothesis is valid, we ran an experiment with a similar experimental paradigm as in Druey and Hübner (2008b). The participants had to perform two tasks on each trial. We manipulated the congruency of the first task by presenting congruent, incongruent, or neutral stimuli, and observed how the congruency of these stimuli affected the RR costs in the second task. Note that in this paradigm the first task corresponds to a previous trial in a standard sequential task-switching paradigm. For simplicity, however, we nevertheless use the term previous-trial congruency even though it refers here to the congruency in the first task on each trial of the experiment. Because task repetitions were not necessary for our objective, we had only task-switch trials.

Different from Druey and Hübner (2008b), we used individual stimulus types (numerals and letters) for each task. The congruency of the stimuli was modulated by presenting irrelevant items in addition to the target item. The irrelevant items could activate the correct response category, the wrong response category, or none of the responses categories. One advantage of using these stimulus types was that we could use univalent stimuli to vary the congruency of the stimuli for first task by presenting irrelevant items that were of the same type as the target item. Accordingly, all items activated their response categories only through the required task and not through the currently irrelevant task, which excludes any alternative explanation of the observed RR effects in terms of task-cueing effects (Steinhauser \& Hübner, 2007).

The crucial comparison in our experiment was that between the RR effects after neutral stimuli and those after incongruent ones. If the absolute-activation hypothesis is correct, then RR costs should be similar in size after responses to neutral and incongruent stimuli. However, if the activation difference determines RR costs, then these costs should be larger after responses to neutral stimuli than after those to incongruent ones.

\subsection{Method}

\subsubsection{Participants}

Twenty-five ( 3 males; mean age 23 years) persons with normal or corrected-to-normal vision participated in the experiment. The participants were students of the Universität Konstanz and were paid $8 € / \mathrm{h}$. Four participants $(16 \%)$ were replaced because of their exceptionally long mean response times or high error rates in the second task (more than 2 standard deviations above the group mean).

\subsubsection{Apparatus}

The stimuli were presented on a 19-inch color monitor with a resolution of $1280 \times 768$ pixels and a refresh rate of $60 \mathrm{~Hz}$. A PC controlled stimulus presentation and response registration. Participants responded with the index and middle fingers of their right hand by pressing one of two mouse buttons.

\subsubsection{Stimuli}

Relevant stimulus items comprised letters ( $G, K, M, R, A, E, O, U$ ), and numerals $(2,4,6,8,1,3,7,9)$. Furthermore, there was a neutral item (star: *) that was unrelated to any task. The stimulus arrays S1 and S2 for Task 1 and Task 2, respectively, were composed of three items. One item was displayed at the center of the screen and the two other identical items were presented laterally. S1 was always univalent (associated only with one task), i.e. the flanker items were either of the same type as the center item or neutral. With this procedure we could construct 16 different neutral S1, and 64 different congruent and incongruent $\$ 1$, respectively. S2 was always bivalent (associated with two tasks) and incongruent, i.e. center item and lateral items were always of a different type and were mapped to opposite responses. Because we found in a recent study (Grzyb \& Hübner, submitted for publication) larger RR costs for bivalent than for univalent stimuli and also for incongruent S2 than for congruent and neutral S2, we used only rule-incongruent $\mathrm{S} 2$ in the present experiment to increase the effects. ${ }^{1}$ To keep the task as simple as possible, the task-relevant item in S1 was always placed in the middle of the array. However, because the spatial separation of target and distractor items would enable participants to reduce interference by spatial filtering, and because we wanted strong RR effects, we implemented spatial uncertainty for the target item in $\$ 2$. Hence, the task-relevant item in $\$ 2$ could be the center item or the flanker items (for details, see Procedure). Accordingly, there were 128 different S2.The stimulus patterns approximately subtended a visual angle of $5.5^{\circ}$ width and of $2.1^{\circ}$ height. The stimuli were displayed in white on a black background.

\footnotetext{
${ }^{1}$ One might speculate that the use of rule-incongruent $S 2$ allowed participants to switch the response mapping within one task instead of switching between tasks. We will discuss this possibility further in the Discussion of this experiment.
} 
Table 1

Mean response times (in ms) and error rates (in \%) of the first response for the different congruency types of the first stimulus in the two experiments, and mean response times (in $\mathrm{ms}$ ) and error rates (in \%) of the second response depending on the congruency of the first task and the response condition in the two experiments. $\mathrm{RT}=$ response time; $\mathrm{ER}=\mathrm{error}$ rate. Parenthetical values are standard errors of the mean.

\begin{tabular}{|c|c|c|c|c|c|c|c|}
\hline & & \multirow{2}{*}{\multicolumn{2}{|c|}{ First response }} & \multicolumn{4}{|c|}{ Second response } \\
\hline & & & & \multicolumn{2}{|c|}{ Response repetition } & \multicolumn{2}{|c|}{ Response shift } \\
\hline & & $\mathrm{RT}$ & ER & $\mathrm{RT}$ & ER & RT & ER \\
\hline \multirow[t]{3}{*}{ Experiment 1} & Congruent & $693(25)$ & $4.07(0.42)$ & $778(34)$ & $12.8(1.3)$ & $741(29)$ & $4.57(.74)$ \\
\hline & Neutral & $653(22)$ & $4.21(0.54)$ & $816(42)$ & $13.8(1.3)$ & $758(32)$ & $5.03(.80)$ \\
\hline & Incongruent & $737(25)$ & $7.40(0.66)$ & $759(31)$ & $11.5(1.1)$ & $746(31)$ & $4.72(.57)$ \\
\hline \multirow[t]{3}{*}{ Experiment 2} & Congruent & $596(16)$ & $4.48(0.55)$ & $676(19)$ & $15.6(1.2)$ & $630(14)$ & $4.75(.59)$ \\
\hline & Neutral & $583(15)$ & $5.30(0.52)$ & $692(18)$ & $16.8(1.6)$ & $654(15)$ & $4.36(.67)$ \\
\hline & Incongruent & $642(16)$ & $8.62(0.84)$ & $650(16)$ & $12.8(.90)$ & $628(14)$ & $4.80(.78)$ \\
\hline
\end{tabular}

\subsubsection{Procedure}

A trial started with the presentation of a cue in red color for $1000 \mathrm{~ms}$. The cue " $\mathrm{g} / \mathrm{u}$ " [abbreviation for the German words "gerade" (even) and "ungerade" (odd)], indicated the parity judgment, and the cue " $\mathrm{k} / \mathrm{v}$ " [abbreviation for German words "Konsonant" (consonant) and "Vokal" (vowel)], symbolized the consonant/vowel judgment. ${ }^{2}$ After the cue, $\$ 1$ was presented for $200 \mathrm{~ms}$ and was followed by a blank screen. After the response to $\mathrm{S} 1$ the stimulus $\mathrm{S} 2$ for the second task was presented for $200 \mathrm{~ms}$. The next trial started $1000 \mathrm{~ms}$ after the response to $\$ 2$.

Participants were instructed to prepare for the upcoming tasks and to respond as quickly and as accurately as possible. On every trial they first had to respond to the center item of S1 and to perform the judgment indicated by the cue. Then, participants had to switch to the other task and to respond to S2. Because S2 was always bivalent and incongruent, participants had to select the relevant item (center item or flankers of S2) on the basis of the item type. That is, if the first task required, for instance, a parity judgment from the participants, then they had to judge the letter(s) in S2.

The experiment consisted of 8 blocks of 96 trials each. These 96 trials were drawn pseudo-randomly out of the 18432 possible sequences, considering relevant factors such as Task 1 , first response, response alternation, and position of the relevant item in S2. Critically, neutral, congruent and incongruent $\mathrm{S} 1$ were pseudo-randomly mixed so that they had an equal frequency (one third) in each block. The first block was considered as a practice block and not analyzed.

\subsection{Results}

Because the relevant item in S1 could repeat as an irrelevant item in $\$ 2$ only in a subset of conditions, i.e. in response-shifts trials, these trials were excluded from the analysis. Also, for every condition trials with response times larger than 4 standard deviation of the mean were excluded $(<2.5 \%$ of all trials).

\subsubsection{First task}

The mean latencies and the error rates (see Table 1) of responses to the first stimulus were entered into separate one-factor ANOVAs with the within-participant factor congruency (neutral, congruent, incongruent).

The analysis of the latencies revealed a significant congruency effect, $F(2,48)=43.4, p<.001$. As can be seen in Table 1 , responses to neutral stimuli were fastest and those to congruent stimuli slowest (congruent: $693 \mathrm{~ms}$, neutral: $653 \mathrm{~ms}$, incongruent: $737 \mathrm{~ms}$ ). Planned contrasts indicated that all pairwise differences were significant (all $p s<.001$ ).

\footnotetext{
2 There were two reasons for varying the task order randomly. First, we wanted to discourage participants to adapt to the mapping-switching strategy described in Footnote 1 . Second, the current paradigm is more similar to a random task-cueing paradigm usually used in task-switching studies.
}

The analysis of the mean error rates also revealed a significant congruency effect, $F(2,48)=23.9, p<.001$. Most errors were made for incongruent stimuli and least to congruent ones (congruent: $4.07 \%$; neutral: $4.21 \%$; incongruent: $7.40 \%$ ). Planned contrasts indicated that all pairwise differences were significant $(p s<.001)$ except the one between congruent and neutral stimuli, $F(1,24)=0.10, p=.75$.

\subsubsection{Second task}

Only responses after a correct response to $S 1$ were included (Steinhauser \& Hübner, 2006). The mean latencies of the responses to the second stimulus were entered into a two-factor ANOVA with the within-participant factors response (repetition, shift) and previous-trial congruency (neutral, congruent, incongruent).

The analysis revealed a significant main effect of response, $F(1,24)=$ $14.8, p<.001$, with longer latencies for response repetitions ( $784 \mathrm{~ms}$ ) than for response shifts $(748 \mathrm{~ms})$. The main effect of previous-trial congruency was also reliable, $F(2,48)=9.28, p<.001$. Mean response times were smaller after responses to congruent $\$ 1(759 \mathrm{~ms})$ than after those to neutral S1 $(787 \mathrm{~ms}), F(1,24)=6.43, p<.05$, and smallest after responses to incongruent $\mathrm{S} 1(752 \mathrm{~ms}), F(1,24)=4.56, p<.05$. Importantly, the interaction of previous-trial congruency and response was also significant, $F(2,48)=4.43, p<.05$, (see Fig. 2). Planned comparisons showed that $R R$ costs (computed as response repetition minus response shifts) were marginally larger after responses to congruent $\$ 1$ (38 ms) than after responses to incongruent S1 (13 ms), $F(1,24)=3.32, p=.081$. Considering that the prediction of higher RR costs after congruent $S 1$ than after incongruent $S 1$ was a directed hypothesis, the $p$-value can actually be halved (yielding $\mathrm{p}<.05$ ). Moreover, RR costs after responses to incongruent $S 1$ were only marginally significant, $t(24)=1.67, p=.054$, one-tailed. Further comparisons showed that RR costs after responses to neutral S1 $(59 \mathrm{~ms})$ were larger than the costs after responses to incongruent $S 1, F(1,24)=7.08$, $p<.05$, but statistically not distinct from the costs after responses to congruent $S 1, F(1,24)=1.94, p=.18$.

An analogous analysis for the mean error rates of the second response revealed significant effects of response, $F(1,24)=59.8, p<.001$. Response repetitions (12.7\%) produced more errors than response shifts (4.77\%). The main effect of the factor previous-trial congruency was marginally significant, $F(2,48)=2.73, p=.075$. Finally, the interaction between previous-trial congruency and response failed to reach significance, $F(2,48)=1.90, p=.16$.

\subsection{Discussion}

The results show that $R R$ costs were larger after a response to a congruent stimulus than after a response to an incongruent one, which not only replicates previous results (Druey \& Hübner, 2008b), but also generalizes these findings to the present experimental paradigm. However, most important for the current objective is that RR costs were also substantially larger after a response to a neutral stimulus than that after a response to an incongruent stimulus. This data pattern 

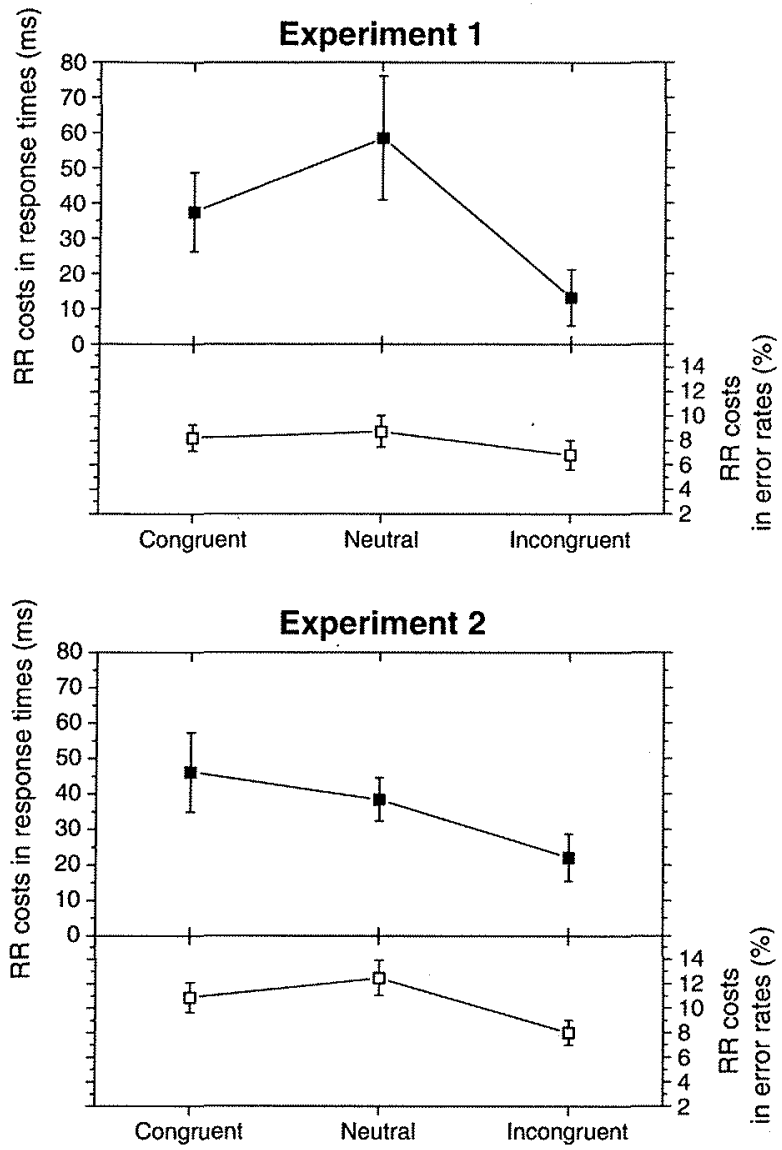

Fig. 2. RR costs in the response times (upper panels) and in the error rates (lower panels) plotted against the congruency of the previous stimulus for Experiments 1 and 2 .

is incompatible with the absolute-activation hypothesis, which predicts that there should be no difference between these two conditions. Thus, our results suggest that it is the activation difference between the alternative response categories that determines the size of response-category inhibition, which, in turn, produces costs if the response has to be repeated.

A potential objection to the present procedure might be that using rule-incongruent $\mathrm{S} 2$ allowed participants to switch response mappings and to respond to the distractor item of $\mathrm{S} 2$, which was always of the same material (letter or numeral) as the target item in S1. Although we think that such a violation of the instructions is unlikely, we cannot rule out that it occurred. However, even if participants performed this way, the rationale of our experiment would still be valid. This is because in either case participants would switch between different sets of S-R mappings. Thus, according to the inhibition account, RR effects would still reflect the pure effect of responsecategory inhibition since the only feature of the previous processing that repeats is the response. Hence, the size of RR costs should still depend on the amount of activation of the response categories corresponding to $\mathrm{S1}$.

An unexpected result was that first-task responses to neutral stimuli were faster than those to congruent stimuli. This could indicate that the activation of the correct response category for neutral stimuli was at least as large as that for congruent stimuli, if not stronger. Hence, one might argue that the activation of the correct response category for neutral stimuli might also have been stronger than that for incongruent stimuli. This would make the rationale of our experiment inadmissible and, therefore, also our conclusion concerning the origin of RR costs.
However, differences in response speed do not only depend on differences in response-category activation but also on differences between other features. In our case, for instance, it is likely that responses to neutral stimuli were relatively fast, because they required little perceptual filtering (Treisman, Kahneman, \& Burkell, 1983). That is, due to the fact that we used only a single symbol, a star, as neutral item, which largely differed from the relevant symbols, perceptual discrimination between the items was relatively easy. This speeded up responding without necessarily increasing the activation of the correct response category. Nonetheless, we cannot definitively rule out that there were also differences in the absolute activation between the correct response category for neutral stimuli and that for incongruent stimuli. Therefore, we conducted a further experiment in which we tried to equalize the difficulty of perceptual filtering across the different congruency conditions.

\section{Experiment 2}

This experiment was similar to the first one except that we used four different symbols as neutral items, instead of one, so that the number of different neutral stimuli was the same as for the other congruency conditions. We expected that this increase in variation will increase the filtering costs for neutral stimuli compared to the previous experiment, such that the responses to neutral stimuli should at least not be faster than those to congruent stimuli. If this manipulation indeed affects only the perceptual stage of processing, then, according to the response-inhibition account, the RR costs after responses to neutral stimuli should be similar to those in the previous experiment. A further methodological difference to the previous experiment was that, because participants' feedback indicated that block length was rather long and exhausting, it was reduced, while maintaining the number of trials.

Concerning our hypotheses, we expected the same order of $R R$ costs, even though the responses to neutral stimuli in the first task should be slower. Thus, we expected further support for the activation-difference hypothesis.

\subsection{Method}

\subsubsection{Participants}

Twenty-six ( 5 males; mean age 22 years) persons with normal or corrected-to-normal vision participated in the experiment. The participants were students of the Universität Konstanz and were paid $8 € / h$. Four participants ( $15 \%$ ) had to be replaced because of exceptionally high mean response times or error rates of the response to the second stimulus (more than 2 standard deviations above the group mean). One further participant was replaced because of a negative congruency effect in both mean response time and error rates of the responses to the first stimulus.

\subsubsection{Apparatus, stimuli, and procedure}

Apparatus, stimuli, and procedure were the same as in Experiment 1 except for the following chances: four symbols (*, \#,\%, and \&) were used as neutral items and the experiment contained 16 blocks à 48 trials. The first two blocks were considered as practice blocks and were not analyzed.

\subsection{Results}

As for the analysis of the first experiment, repetitions of the target item in S1 as the non-target item in S2 were excluded from the data analysis. Also, for every condition trials with response times larger than 3.5 standard deviations of the subject mean were excluded from the analysis ( $<2.5 \%$ of all trials). The results are summarized in Table 1. 


\subsubsection{First task}

Mean latencies of correct responses and mean error rates were entered into separate ANOVAs with the within-participant factor congruency (neutral, congruent, incongruent).

The analysis of the latencies revealed a significant effect, $F(2,50)=$ $81.1, p<.001$. Planned comparisons revealed that responses to neutral stimuli ( $583 \mathrm{~ms}$ ) were faster than those to congruent stimuli ( $596 \mathrm{~ms}), F(1,25)=12.2, p<.01$, and that responses to incongruent stimuli $(642 \mathrm{~ms})$ were the slowest, both $p s<.001$.

The analyses of the error data showed a significant effect of congruency, $F(2,50)=26.9, p<.001$, with responses to congruent (4.48\%) and neutral stimuli (5.30\%) being more accurate than responses to incongruent stimuli (8.62\%). Planned comparison revealed that responses to congruent stimuli were more accurate than responses to neutral stimuli, $F(1,25)=5.61, p<.05$, which indicates a speedaccuracy tradeoff.

\subsubsection{Second task}

The mean latencies of correct responses were entered into a two-factor ANOVA with the within-participant factor previous-trial congruency (neutral, congruent, incongruent) and response (repetition, shift). The analysis showed a significant main-effect of previoustrial congruency, $F(2,50)=23.6, p<.001$. Mean response times after responses to incongruent $S 1$ were shortest $(639 \mathrm{~ms})$, followed by responses times after responses to congruent $S 1(653 \mathrm{~ms})$ and by latencies after responses to neutral S1 were slowest $(673 \mathrm{~ms})$. Also, response repetitions were slower $(673 \mathrm{~ms})$ than response shifts $(637 \mathrm{~ms}), F(1,25)=32.5, p<.001$. However, these results were qualified by a two-way interaction of both factors, $F(2,50)=3.39, p<.05$. As in Experiment 1, we report effects of previous-trial congruency in terms of RR costs to examine this interaction. Planned comparisons revealed that costs were larger after responses to congruent $(46 \mathrm{~ms})$ than after responses to incongruent $51(22 \mathrm{~ms}), F(1,25)=6.15$, $p<.05$. RR costs after responses to neutral $51(38 \mathrm{~ms})$ were marginally larger than after responses to incongruent $51, F(1,25)=3.70$, $p=.066$. Finally, RR costs did not differ between the performance after responses to neutral and that after responses to congruent $\mathrm{S} 1$, $F(1,25)=0.59, p=.45$.

The corresponding analysis of the mean error rates of the second response revealed significant effects of response, $F(1,25)=116$, $p<.001$, and of previous-trial congruency, $F(2,50)=3.90, p<.05$. Response repetitions (15.1\%) were associated with increased error rates compared to response shifts (4.64\%). Also, accuracy after responses to incongruent $S 1(8.81 \%)$ was increased compared to that after responses to congruent or neutral $\mathrm{S} 1(10.2 \%$ and $10.6 \%$, respectively). Importantly, these effects were qualified by an two-way interaction between the two factors, $F(2,50)=6.20, p<.01$. Planned comparisons showed that $R R$ costs were larger after responses to congruent $(10.9 \%)$ than after those to incongruent $S 1(8.02 \%), F(1,25)=$ $5.02, p<.05$. Furthermore, the difference between $\mathrm{RR}$ costs after responses to neutral $\mathrm{S} 1$ (12.5\%) and those after responses to incongruent S1 was also significant, $F(1,25)=12.1, p<.01$. Finally, although $R R$ costs were numerically larger after responses to neutral $S 1$ than those after responses to congruent $\mathrm{S1}$, this difference was statistically not significant, $F(1,25)=1.55, p=.22$.

\subsection{Discussion}

As expected, the crucial result of Experiment 1 could be replicated. $R R$ costs were larger after responses to congruent and neutral stimuli (S1) than after responses to incongruent stimuli. For the performance after responses to neutral stimuli this held for the error rates, whereas for that after responses to congruent stimuli this was true for the response times as well as for the error rates. Again, as in Experiment 1 , there were no significant RR cost differences between the conditions with congruent and neutral previous stimuli.
Concerning the performance in the first task (see Table 1) responses to neutral stimuli were again faster than responses to congruent ones. Here, however, this can be attributed to a speed-accuracy trade off, because their accuracy was significantly lower. Thus, compared to Experiment 1, responses to neutral stimuli decreased significantly in speed $(F(1,49)=7.14, p=0.01)$ while the corresponding RR costs were of comparable size $(F(1,49)=1.19)$. This demonstrates that the response time differences for the neutral stimuli between the experiments can be attributed to different perceptual filtering demands and not to differences in the absolute activation of the correct response category. If the absolute response category activation would have been lower in Experiment 2, compared to Experiment 1, then we should also have observed smaller RR costs after responses to neutral stimuli. Because this was not the case, we can rule out that neutral stimuli produced larger absolute activation of the correct response category than incongruent stimuli.

\section{General discussion}

The aim of the present study was to examine the origin of the previous-trial response activation effect, i.e. the modulation of $R R$ costs by the response-category activations on the previous trial. Preceding research indicates that after the execution of a response the corresponding response category is inhibited and that the amount of this inhibition depends on the amount of response-category activation on the previous trial (Druey \& Hübner, 2008b). However, what remained open was the specific property of response-category activation that is crucial in this respect. Therefore, in the present study we contrasted two possible hypotheses: the absolute-activation hypothesis and the activation-difference hypothesis. The first hypothesis states that the absolute activation of the correct response category is responsible for the size of response inhibition, whereas the second hypothesis assumes that the difference in activation between the correct response category and its alternative is crucial. To test which of the two hypotheses is valid, we conducted two experiments in which response-category activation was modulated by varying irrelevant stimulus features of a first task in a two-task paradigm. More specifically, the stimuli in the first task could be congruent, neutral, or incongruent.

The critical comparison was that between the RR costs after responses to neutral stimuli and those after incongruent stimuli. Because the task-irrelevant features of both stimulus types do not affect the correct response category, the absolute activation of the correct response category should be similar for both stimulus types. In contrast, incongruent stimuli also activate the opposite response category, which is not the case for neutral stimuli. Therefore, the activation difference between the alternative response categories should be larger for neutral than for incongruent stimuli. As the data of both experiments show, RR costs were larger after responses to neutral than after those to incongruent stimuli. These results strongly support the activation-difference hypothesis and cannot be explained by differences between the absolute activations of the correct response category.

Although the condition with congruent stimuli in the first task was less important for testing the two hypotheses, the corresponding performance should be consistent. Surprisingly, though, in Experiment 1 responses to neutral stimuli were faster than those to congruent ones, but nevertheless produced similar RR costs. This seemed to contradict both of our hypotheses. However, because we used only a single and distinct symbol as irrelevant neutral item, we supposed that the increased response speed for neutral stimuli relative to that for congruent stimuli had been due to reduced perceptual filtering costs rather than to increased response-category activation. To test this conjecture, we used four different neutral symbols in Experiment 2. As expected, this measure reduced the performance to neutral stimuli without reducing RR costs, which confirmed our supposition that 
small perceptual filtering costs were responsible for the fast responses to neutral stimuli in Experiment 1.

Interestingly, the variation in speed of the responses to neutral stimuli between the two experiments is also informative with respect to another hypothesis. One could have speculated that RR costs depend directly on the response time on the previous trial. However, the fact that the responses times for neutral stimuli but not the corresponding RR costs varied significantly across our two experiments, demonstrates that such a hypothesis does not hold. Rather, our data indicate that only processes involved in response selection determine the size of subsequent response-category inhibition.

The result that $R R$ costs did not differ after responses to congruent stimuli compared to those after responses to neutral stimuli could indicate that response-category activations were rather similar for neutral and congruent stimuli. This view is in line with LRP studies that failed to find coactivation of responses to redundant visual signals (Mordkoff, Miller, \& Roch, 1996; but see, Mattler, 2003; Smid, Mulder, \& Mulder, 1990). However, this would not undermine the interpretation of the present results regarding the two alternative hypotheses. If the absolute-activation hypothesis would be true, then one would still predicted similar RR costs after responses to neutral than after those to incongruent stimuli, which was not the case. Thus, the possibility that congruent stimuli did not produce a larger activation of the correct response category than neutral ones is consistent with the activation-difference hypothesis.

Increasing the number of symbols for constructing neutral stimuli in Experiment 2 reduced the performance for these stimuli, relative to that in Experiment 1, but it was still not poorer than that for congruent stimuli. Although similar performance for neutral and congruent stimuli is not unusual (Eriksen \& Eriksen, 1974), in view of the present data one could argue that response conflict rather than the difference in response-category activation was the crucial factor. Nonconflicting stimuli (neutral and congruent ones) could have produced a certain amount of RR costs, whereas the costs would be reduced after conflicting stimuli (incongruent ones) by some kind of conflict adaptation (Botvinick, Braver, Barch, Carter, \& Cohen, 2001; Brown, Reynolds, \& Braver, 2007). However, such a mechanism would also predict an adaptation of performance on response shift trials. That is, response-shift performance after responses to incongruent stimuli should be better than response-shift performance after responses to congruent stimuli (Altmann, 2011). But, as can be seen in Table 1, this was not the case.

Furthermore, there are data showing that RR costs also vary within non-conflicting conditions. In a recent study, Koch, Schuch, $\mathrm{Vu}$, and Proctor (2011) modulated response-category activation by spatial response discriminability, i.e. by changing the distance between the response keys. With more easily discriminable response codes the corresponding activation differences should be larger, which, according to the activation-difference hypothesis should increase the RR effects. This was exactly what Koch et al. have found. When the spatial separation of the response keys was large and, thus, the overlap between the response categories was small, RR costs on task-switch trials were larger than when the spatial separation was small. Because stimulus information was the same in both conditions, this result cannot easily be explained by conflict adaptation, but is consistent with the activation-difference hypothesis.

\subsection{Conclusions}

To sum up, the present results further support the responseinhibition account of RR effects (Hübner \& Druey, 2006). Response categories are inhibited after the execution of the corresponding response in order to prevent perseveration errors. Moreover, the amount of this inhibition depends on the activation difference between the alternative response categories. The larger this difference, the greater the RR costs on the next trial. Future studies should use measures of response-category activation, such as the lateralized readiness potential (LRP, Gratton, Coles, Sirevaag, Eriksen, \& Donchin, 1988), to investigate the relation between the activation difference between response categories and the size of subsequent $R R$ costs more directly.

\section{Acknowledgments}

This research was supported by a grant ( $\mathrm{Hu} 432 / 9$ ) from the Deutsche Forschungsgemeinschaft (DFG) to the co-author.

\section{References}

Altmann, E. M. (2011). Testing probability matching and episodic retrieval accounts of response repetition effects in task switching. Joumal of Experimental Psychology: Learning, Memory, and Cognition, 37, 935-951.

Botvinick, M. M., Braver, T. S., Barch, D. M., Carter, C. S., \& Cohen, J. D. (2001). Conflict monitoring and cognitive control. Psychological Review, 108, 624-652.

Brown, J. W., Reynolds, J. R. \& Braver, T. S. (2007). A computational model of fractionated conflict-control mechanisms in task-switching. Cognitive Psychology, 55, 37-85.

Cooper, S., \& Mari-Beffa, P. (2008). The role of response repetition in task switching. joumal of Experimental Psychology. Human Perception and Performance, 34, 1198-1211. Druey, M. D., \& Hübner, R. (2008a). Effects of stimulus features and instruction on response coding, selection, and inhibition: Evidence from repetition effects under task switching. The Quarterly Journal of Experimental Psychology, 61, 1573-1600.

Druey, M. D. \& Hübner. R. (2008b). Response inhibition under task switching: Its strength depends on the amount of task-irrelevant response activation. Psychological Research/Psychologische Forschung, 72, 515-527.

Eriksen, B. A., \& Eriksen, C. W. (1974). Effects of noise letters upon the identification of a target letter in a nonsearch task. Perception \& Psychophysics, 16, 143-149.

Gratton, G., Coles, M. G. H., Sirevaag, E. J., Eriksen, C. W., \& Donchin, E. (1988). Pre- and poststimulus activation of response channels: A psychophysiological analysis. Journal of Experimental Psychology. Human Perception and Performance, 14, 331-344.

Grzyb, K.R., Hubner, R. (submitted for publication). Response-repetition costs under task switching: Their modulation by bottom-up processes.

Hommel, B. (2010). The Simon effect as tool and heuristic. Acta Psychologica, doi: $10.1016 / j$.actpsy.2010.04.011.

Hübner, R., \& Druey, M. D. (2006). Response execution, selection, or activation: What is sufficient for response-related repetition effects under task shifting? Psychological Research/Psychologische forschung, 70, 245-261.

Kiesel, A., Steinhauser, M., Wendt, M., Falkenstein, M., Jost, K., Philipp, A. M., et al. (2010). Control and interference in task switching - A review. Psychological Bulletin $136,849-874$

Kleinsorge, T. (1999). Response repetition benefits and costs. Acto Psychologica, 103, 295-310.

Koch, I. Schuch, S., Vu, K. P., \& Proctor, R. W. (2011). Response-repetition effects in task switching-Dissociating effects of anatomical and spatial response discriminability. Acta Psychologica, 136, 399-404.

Mattler, U. (2003). Delayed flanker effects on lateralized readiness potentials. Experimental Brain Research, 151, 272-288.

Mattler, U. (2005). Flanker effects on motor output and the late-level response activation hypothesis. The Quarterly Joumal of Experimental Psychology, 58, 577-601.

Meiran, N. (2000). Modeling cognitive control in task-switching. Psychological Research/Psychologische Forschung, 63, 234-249.

Mordkoff, J. T., Miller, J., \& Roch, A. -C. (1996). Absense of coactivation in the motor component: Evidence from psychophysiological measures of target detection. Journal of Experimental Psychology. Human Perception and Performance, 22, 25-41.

Ratcliff, R. (1978). A theory of memory retrieval, Psychological Review, 85, 59-108.

Ratcliff, R., \& Rouder, J. N. (1998). Modeling response times for two-choice decisions. Psychological Science, 9, 347-356.

Rogers, R. D., \& Monsell, S. (1995). Costs of a predictable switch between simple cognitive tasks. Journal of Experimental Psychology. General, 124, 207-23!

Schuch, S., \& Koch, l. (2004). The costs of changing the representation of action: Response repetition and response-response compatibility in dual tasks. Journal of Experimental Psychology. Human Perception and Performance, 30, 566 582 .

Simon, J. R. (1969). Reactions toward the source of stimulation. Joumal of Experimental Psychology, 81, 174-176.

Smid, H. G., Mulder, G., \& Mulder, L. J. (1990). Selective response activation can begin before stimulus recognition is complete: A psychophysiological and error analysis of continuous flow. Acta Psychologica, 74, 169-201.

Steinhauser, M. \& Hübner, R. (2006). Response-based strengthening in task shifting: Evidence from shift effects produced by errors. Journal of Experimental Psychology. Human Perception and Performance, 32, 517 -534.

Steinhauser, M. \& Hübner, R. (2007). Automatic activation of task-related representations in task shifting. Memory \& Cognition, 35, 138-155.

Treisman, A., Kahneman, D., \& Burkell, J. (1983). Perceptual objects and the cost of filtering. Perception \& Psychophysics, 33, 527--532. 